\title{
Expertise, Experience, and Effectiveness
}

\section{Daryl Siedentop and Eitan Eldar}

In his 1986 AERA Presidential Address, David Berliner (1986) elevated the issue of teacher expertise to a lofty position in teaching research, where it has remained securely. Bloom's (1986) work had led in that direction, an almost predictable extension of an era focused on teacher effectiveness. Kennedy (1987) recently reviewed the various theoretical approaches to expertise, and the topic has been no stranger to teaching researchers in physical education (Housner \& Griffey, 1985). Given our study of effective elementary specialists, who varied in degrees of experience, it seemed useful to consider them also in terms of expertise.

Berliner's seminal article lays out convincingly the reasons for studying expert teachers, yet seems less than satisfactory when specifying criteria for identifying expert teachers. One is confronted immediately with the sticky task of distinguishing between effectiveness and expertise, which is even more confounded when experience is factored into the equation.

The first article in this monograph contains evidence that justifies the use of the term "effective" when talking about the seven teachers in our study. The start-of-the-school-year study (see article by Fink \& Siedentop) gave us an immediate indication that our assumptions of effectiveness in choosing teachers to include in the study had been warranted. As we had suspected, the effectiveness of the experienced teachers looked a bit different than that of the intermediate and 1st-year teachers. All seven teachers established routines and set clear behavioral parameters for their children. The function of experience was not so much in what they accomplished-predictability, expectations for on-task behavior, routinization of managerial tasks-but in how they accomplished it. The more experienced teachers paced events more smoothly, followed up more deliberately on important behavioral specifications, and utilized content more imaginatively to teach routines.

One way to conceptualize the differences between the 1st-year teachers and the veterans was the ease with which they accomplished their start-of-school-year goals. In this study we saw differences that seemed strikingly similar to those reported by Shulman (1987):

What these studies show is that the knowledge, understanding, and skill we see displayed haltingly, and occasionally masterfully, among beginners are often demonstrated with ease by the expert. (p. 5) 
This "ease" with which veteran teachers accomplished their goals is no doubt also similar conceptually to Bloom's (1986) notion of automaticity. The 1st-year teachers accomplished exactly the same goals. We have no doubt that they were highly effective as beginning teachers! Yet they had not yet achieved automaticity, and there was no reason to expect them to have displayed that characteristic.

This gave us confidence that 1st-year teachers can be effective and that experience on the job can serve to enrich and deepen that effectiveness. But what of expertise? Were our veterans to be considered expert solely because they were effective and had extensive experience? The terms effectiveness and expertise have been used interchangeably too often. It is our sense that if expertise simply means experienced effectiveness, then that is what it should be called. There is no doubt a great deal to be learned from studying experienced effectiveness. How does beginning effectiveness get refined? What processes sharpen effectiveness? What processes dull it? What factors continue to motivate effective teachers to persist in improving their teaching year after year?

Yet the conceptual and operational distinctions between effectiveness and expertise are intriguing and deserve to be explored. We had this exploration in mind when this study began, and we pursued it whenever we interviewed people who knew the subjects, or whenever we were able to address the distinction.

\section{Some Views of Significant Others}

As we considered the topic of effectiveness/expertise throughout the year, we asked as many persons as we could, who might have a point of view that would help to illuminate our study, their views on this distinction. Here are some results from those discussions:

1. Twelve student teachers in a seminar were asked to characterize and differentiate effectiveness and expertise, and then to choose from a selected list six indicators for each. Generally they chose indicators for effectiveness in terms of teacher performance and student outcomes (e.g., manages so as to produce high ALT, establishes routines, anticipates problems, achieves goals) while they chose indicators for expertise in terms of knowledge, ability to articulate, and professional pride (e.g., knows theory of teaching, can explain subject matter, intensive planning).

2. Several principals of our teachers and heads of PTAs were asked to make the distinction, and they did so reliably. Here too, effectiveness was described in terms of teacher performance while expertise tended to reflect the capability to articulate and rationalize performance. It was clear that effectiveness was perceived as knowing how to do, while expertise was perceived as knowing about.

3. Several persons from other fields (all of whom we believed qualified as experts in those fields) were asked and, while their answers tended to be articulated in terms of their fields, we were able to discern some commonalities. The first common theme we would label as technical virtuosity (a term used by a cello player). Experts had to be skilled to the highest degree possible. Second, each suggested that the application of technical virtuosity was highly contextual, that is, the technical skill had to be applied to the specific situation in such a way as to show knowledge of the context (to play the "color" of the music as well 
as the notes, to design a house that respects the client and the site, to use your technical craft to build a crown or a bridge that completely satisfies the patient's needs). Third, each person mentioned in some way that the expert performs these feats reliably and consistently.

Among these three groups we had found a rather serious disagreement. The student teachers, principals, and PTA members tended to describe expertise as a cognitive component, the ability to articulate and rationalize one's own effectiveness, a view that comes very close to what Kennedy (1987) described as the "deliberate action" theory of expertise. The experts from other fields tended to focus more on performance and outcomes than on rationalizations for those performances or outcomes. Performance here, it should be noted, goes well beyond the "technical skills" theory of expertise reviewed and dismissed by Kennedy.

\section{The Views of the Seven Subjects}

At the debriefing with our subjects at the end of the study, the topic of effectiveness/expertise was explored at length. The issue was described briefly and a summary was presented of the views obtained. The teachers in our study did not relate easily to the distinction. Indeed, if they had a summary conclusion it would have been that expertise was merely an extension of effectiveness, a difference in degree, not kind. This became clear as they talked about the two qualities. There was talk about top-notch teachers who designed innovative lessons and did so on a regular basis. There was also mention of being able to discern quickly when things weren't going as planned, and the ability to make slight adjustments "on the fly" to bring a lesson back to its intended course or to slightly alter that course. Mike talked about his growing confidence in organizing and transitioning children and keeping them task oriented, but he also added, "I would have to greatly refine my skills and individualize before I could be an expert.",

It was clear that each of the seven felt more expert in those activities in which they had strong backgrounds. There was an obvious difference in how they saw themselves as teachers based on their knowledge of and experience in the activity. Chris said, "when it comes to gymnastics, I know exactly how I want them to do the skills," a point of view that was clearly understood by the other teachers. Kelley talked about her inability to teach strategy in invasion games, because of her lack of participatory experience in those activities beyond that which she received in her teacher education program.

During the discussion it became clear that contextual differences were an important factor in effectiveness/expertise. None of the teachers felt they could perform effectively if the context of teaching was changed dramatically. Bobbie suggested that across her career she had grown considerably more effective with primary grade children than with intermediates. When asked about their potential for being effective in a more difficult, inner city context, the most common response from the teachers was, "I wouldn't do very well."

These seven teachers tended to view expertise as an extension of effectiveness, a difference in degree, not in kind. They also viewed their own effectiveness, and that of others, as highly contextual. Only when the discussion shifted to subject matter knowledge was a factor other than teaching skill entered into the equation. 


\section{Expertise: Our Emerging View}

After studying seven effective teachers for one school year, discussing teaching expertise with many persons, reviewing the literature, and discussing the issue among ourselves, a coherent view of expertise began to emerge and we then began to apply that view to our seven teachers. Although our views at this point, and their application to these subjects, are highly speculative, we do believe that expertise in teaching exists and that effectiveness and experience are necessary but not sufficient conditions for demonstrating it.

We believe expertise in teaching is highly specific to context and subject matter. In that sense, we do not believe it useful to talk about the expert teacher but rather the expert teacher of gymnastics or poetry or algebra. We also believe that expertise is typically developed within context as a function of experience, and probably does not generalize readily to other contexts; for example, an expert teacher of soccer in a suburban middle school might not be expert in a city elementary school.

It is our view that expertise is to be found at the nexus of skillful teaching and thorough command of the subject matter. The expert teacher combines high levels of teaching skill (the technical virtuosity component) with high levels of subject matter competence, both applied through experience to a particular context. In this sense, Shulman's (1986) pedagogical content knowledge is the most appropriate domain for understanding expertise.

We believe expertise is performance oriented. All of the examples of expertise cited by Berliner (1986) and Bloom (1986) were clearly performers. There may be instances when the relevant performance is primarily cognitive, for example the expert literary critic, but we do not believe that the essence of expertise is in the ability to articulate or rationalize performance but rather in the performance itself. Expert teachers perform differently than effective teachers.

Experience is a necessary but not a sufficient condition for expertise. This is because expertise is probably developed contingently over long periods of time within a specific context. The impressive performances of our 1st-year teachers give us hope that effectiveness can result immediately from good teacher education. But training is primarily the establishment of rule-governed behavior (when in this situation, do that, because of these reasons) with only small amounts of practice (wherein direct contact with the contingencies can shape the behavior). It is our sense that not enough is known about expertise to teach it in the form of rule-governed behavior. It is more likely that direct contact with contingencies over a long period of time is necessary to shape expertise.

If expertise exists, it lies at the nexus of highly skilled teaching and mastery of a subject matter. We believe that many physical education teachers can be highly effective with what we would call basic competence in an activity area. Thus, it is our view that mastery of a subject matter is not a necessary condition for teaching effectiveness. Many teachers, including our seven subjects for most of the activities in their programs, teach quite effectively with moderate levels of competence in most of the activity units in their programs. Meta-analyses on the relationship between teacher knowledge of subject matter and student achievement support this view (Berliner, 1984). Expertise demands, however, that the teacher be a master of the subject area as well as a highly skilled teacher. 
It is also our view that physical education teachers would rank much lower in subject matter competence than would counterparts in mathematics, science, foreign language, and music. This results, we believe, from an undergraduate curriculum that focuses much more strongly on knowing about their subject matter from various disciplinary perspectives (history, psychology, sociology, biomechanics, and physiology of sport and exercise) than direct experience in learning the subject matter itself (soccer, gymnastics, handball, tennis, etc.). Thus we believe one is less likely to find expert physical education teaching in schools than in other subject matters. If and where one does find it, it would be our assumption that the subject matter mastery needed for forging the expertise was acquired outside the teacher preparation program, typically through a long history of participation and/or coaching.

With this emerging view, then, how did our teachers look? Remember, we have argued that they were all effective and we had some evidence that their effectiveness was sharpened with experience. It is our sense that the expertise evident in our teachers was primarily a function of a high degree of subject matter competence blended with the experience of having taught that subject matter to children for many years, but that it was reasonably rare even in this select group.

Chris had a long performance and coaching history in gymnastics, and we believe her to be an expert teacher of children's gymnastics. We believe Bobbie shows expertise in teaching movement skills to very young children. She had studied with four different nationally prominent movement educators as an undergraduate and master's student, and had tried the "formula" approaches to movement education only to discard them as she developed her own unique orientation to early skill development. Gary shows expertise in teaching adventure skills such as climbing and rappelling. He had not only done these skills himself but had long ago visited Project Adventure and trained himself in adventure education. He had honed this expertise not only with the children in his school program but also as director of a local adventure education camp. These were our three veteran teachers, and each was expert in one aspect of a curriculum while still being highly effective in most other aspects of the curriculum.

We saw some signs that other teachers were clearly on the way toward expertise in certain areas. They simply needed time and continued effort. But none of the teachers had the subject matter mastery to be expert in a large part of the elementary curriculum, even though all possessed the effective technical skills and some had sufficient experience. Thus, at least for these physical education specialists, it was the subject matter mastery that was the critical element in expertise. It also needs to be noted that lack of expertise, as defined here, did not prevent any of these teachers from providing a thoroughly sound physical education program for the children in their schools.

\section{Expertise: A Behavioral Interpretation}

Almost all of the expertise literature in teaching is grounded in cognitive theory. We thought it might be useful to present a behavioral interpretation.

1. Expertise is primarily a matter of fine stimulus control. Experts are said to "see things" that nonexperts don't see and to have metacognitive capabilities. Behaviorally, this means they have developed considerably finer discriminations. The finer discriminatory capability leads to greater response differentiation, that 
is, they respond differently to slight changes in the context. Sizer (1984) refers to this as the subtle quality of judgment, a quality that he says is the key to effective teaching.

2. Experts clearly respond more quickly (they have shorter latencies), which Bloom (1986) calls automaticity. Behaviorally, this means that they make discriminations based on information that occurs earlier than for a novice, and that they have had sufficient practice in similar situations to have reduced response latencies. This capability to discriminate early and respond quickly allows them to anticipate, to "look ahead." It is in this area of expertise that the notion of reflection needs to be addressed more seriously, because it is clear that experts, if they do indeed reflect while performing, do it very quickly.

3. Experts clearly have larger response repertoires. They have learned finer discriminations for that context-specific subject matter and have acquired differentiated responses for those discriminations; for example, they not only see things differently but they have more ways to respond to what they see. Our sense is that this aspect of expertise is probably more subject-matter dependent than teaching-skill dependent, although it clearly falls within Shulman's (1986) notion of pedagogical content knowledge.

4. Experts clearly are under control of more complex elements of the stimulus field than are novices. Our cellist said that experts play the "color" of the music rather than just the notes. What this means is that the expert has studied the composer, the context in which the composition was rendered, and the interpretation desired. Behaviorally, these "understandings" function as setting events (Cooper, Heward, \& Heron, 1987). The setting event, once acquired, affects all discriminations that follow. Thus the expert makes discriminations based on stimulus control that goes well beyond the immediate stimulus field. Setting events, which involve prior knowledge of the activity area and those who learn within it, affect the discriminations made based solely on immediate information.

5. Expert teachers are what we call "'plan independent"' (see article by Stroot \& Morton). They did plan carefully at some time for what they do, but their dayto-day performance is plan independent. Behaviorally, the plan functions as a setting event while the execution of that plan is more under the direct control of events during the class. But remember, the setting event affects how the teacher interprets what goes on during the class! The novice is more likely to be plan dependent, and the plan does not represent the rich experience that it does for the veteran; for example it is less likely to be an important setting event. The teaching behavior of the novice is more under control of the plan and is therefore less sensitive to the ongoing events of the class. It is our belief that this particular behavioral phenomenon is a characteristic of effective teachers, not only of expert teachers.

6. Experts probably can articulate and rationalize their performance differently than nonexperts, but that is more a function of the verbal community that has accompanied the development of their expertise than of the expertise itself. Expertise typically develops within a community (mentors, apprentices, novices) in which one learns to talk about one's growing expertise while it develops. For example, as cellists learn to be expert players, they also learn to talk about their technique, the compositions they play, and the interaction between the two. Many performances are viewed by their mentors and followed by critiques, thus improving both the performance capability and the ability to discuss the performance 
articulately. This kind of dual capability also seems to accompany the development of most sport expertise. Interestingly, it is probably less true for teaching expertise, which may be shaped up in relative solitude without a constantly interacting, critical community within which the capacity to articulate one's performance might be developed simultaneously with the performance capability.

\section{Conclusion}

Expertise does exist in teaching. Effectiveness and experience are necessary but not sufficient conditions for achieving it. Expertise is specific to subject matter and context. Thus the expert teachers in our study were expert in particular activities with particular age groups. We got no sense that this expertise would readily transfer, although we suspect that if shifted to a very different context the experts would have an easier time than those who were less than expert. In physical education teaching, it appears to us that lack of subject matter competence is more likely to retard the development of expertise than any other factor.

Expertise can be explained behaviorally. The expert is under considerably more complex stimulus control, with a larger, more highly differentiated response repertoire, and with stronger control from setting events relative to the subject matter as applied to a particular context.

Do we need more experts in physical education teaching? It would be nice to have them. The way to get them is to provide much stiffer preparation in subject matter (e.g., gymnastics, soccer, movement). On the other hand, it is clear that effectiveness is within the reach of almost all who prepare to become teachers of physical education, and it ought to be better ensured as a first step. 